\title{
A Survey of Bohmian Mechanics
}

\author{
K. Berndl, M. Daumer, and D. Dürr \\ Mathematisches Institut der Universität München, \\ Theresienstraße 39, 80333 München, Germany \\ S. Goldstein \\ Department of Mathematics, Rutgers University, \\ New Brunswick, NJ 08903, USA \\ N. Zanghì \\ Dipartimento di Fisica, Università di Genova, Sezione INFN Genova, \\ Via Dodecaneso 33, 16146 Genova, Italy
}

Bohmian mechanics is the most naively obvious embedding imaginable of Schrödinger's equation into a completely coherent physical theory. It describes a world in which particles move in a highly non-Newtonian sort of way, one which may at first appear to have little to do with the spectrum of predictions of quantum mechanics. It turns out, however, that as a consequence of the defining dynamical equations of Bohmian mechanics, when a system has wave function $\psi$ its configuration is typically random, with probability density $\rho$ given by $|\psi|^{2}$, the quantum equilibrium distribution. It also turns out that the entire quantum formalism, operators as observables and all the rest, is a consequence of Bohmian mechanics.

03.65. Bz

August 2, 1994

\section{BOHMIAN MECHANICS IN A NUTSHELL}

Suppose that when we talk about the wave function of a system of $N$ particles, we seriously mean what our language conveys, i.e., suppose we insist that "particles" means particles. If so, then the wave function cannot provide a complete description of the state of the system; we must also specify its most important feature, the positions of the particles themselves!

Suppose, in fact, that the complete description of the quantum system - its state - is given by

$$
(Q, \psi)
$$

where $Q=\left(\mathbf{Q}_{1} \ldots \mathbf{Q}_{N}\right) \in \mathbb{R}^{3 N}$, with $\mathbf{Q}_{k}$ the positions of particles, and $\psi=\psi(q)=\psi\left(\mathbf{q}_{1} \ldots \mathbf{q}_{N}\right)$ is the wave function. Then we shall have a theory once we specify the law of motion for the state $(Q, \psi)$. The simplest possibility is that this motion is given by first-order equations - so that $(Q, \psi)$ is indeed the state in the sense that its present specification determines the future. We already have an evolution equation for $\psi$, i.e., Schrödinger's equation,

$$
i \hbar \frac{\partial \psi}{\partial t}=-\sum_{k=1}^{N} \frac{\hbar^{2}}{2 m_{k}} \Delta_{k} \psi+V \psi .
$$

According to what we have just said we are looking for an evolution equation for $Q$ of the form

$$
\frac{d Q}{d t}=v^{\psi}(Q)
$$

where $v^{\psi}=\left(\mathbf{v}_{1}^{\psi} \ldots \mathbf{v}_{N}^{\psi}\right)$. Thus the role of $\psi$ is to choreograph a motion of particles through the vector field on configuration space that it defines,

$$
\psi \rightarrow v^{\psi} .
$$

But how should $v^{\psi}$ be chosen? A specific form for $v^{\psi}$ emerges by requiring space-time symmetry - Galilean and time-reversal invariance (or covariance), and "simplicity" [1]:

For one-particle system we find

$$
\mathbf{v}^{\psi}=\frac{\hbar}{m} \operatorname{Im} \frac{\boldsymbol{\nabla} \psi}{\psi},
$$

and for a general $N$-particle system

$$
\mathbf{v}_{k}^{\psi}=\frac{\hbar}{m_{k}} \operatorname{Im} \frac{\boldsymbol{\nabla}_{k} \psi}{\psi} .
$$

We've arrived at Bohmian mechanics, defined by (13 ) for a nonrelativistic system (universe) of $N$ particles, without spin. This theory, a refinement of de Broglie's pilot wave model, was found and compellingly analyzed by David Bohm in 1952 [2 6, 1. 7 10]. Spin, as well as Fermi and Bose-Einstein statistics, can easily be dealt with and in fact arise in a natural manner [11,2,12 14].

Let us briefly mention how to incorporate spin into Bohmian mechanics.. Note that on the right-hand side of the equation for the velocity field the $\boldsymbol{\nabla}$ is suggested by rotation invariance, the $\psi$ in the denominator by homogeneity, the "Im" by time-reversal invariance, and the constant in front is precisely what is required for covariance under Galilean boosts. Rotation invariance requires in particular that rotations act on the value space of the wave function. But the latter action is rather inconspicuous for spinless particles. The simplest nontrivial 
(projective) representation of the rotation group is the 2-dimensional "spin $\frac{1}{2}$ " representation. This representation leads to a Bohmian mechanics involving spinorvalued wave functions for a single particle (and spinortensor-product-valued wave function for many particles). Beyond the fact that the wave function now has a more abstract value space, nothing much changes from our previous description: The wave function evolves according to a Hamiltonian that contains the Pauli term, for a single particle proportional to $\mathbf{B} \cdot \boldsymbol{\sigma}$, which represents the coupling between the "spin" and an external magnetic field B. The configuration evolves according to the natural extension of the velocity field to spinors, obtained, say, by multiplying both the numerator and denominator of the argument of "Im" on the left by $\psi^{*}$ And interpreting the result for the case of spinor values as a spinor-innerproduct:

$$
\mathbf{v}^{\psi}=\frac{\hbar}{m} \operatorname{Im} \frac{\psi^{*} \nabla \psi}{\psi^{*} \psi}
$$

A remark on Bose-Fermi statistics: According to orthodox quantum mechanics, the very notion of indistinguishable particles seems to be grounded on the nonexistence of particle trajectories and on the practical impossibility of distinguishing identical particles at two different times. This might lead to the expectation that it should be quite problematical to incorporate the description of indistinguishable particles into Bohmian mechanics. However, this is not so. Indeed, the usual symmetry conditions on the wave function arise naturally when the Bohmian approach is applied to systems of indistinguishable particles. Moreover, when spin is taken into account, the fact that the intermediate statistics (the so called parastatistics) are to be excluded turns out to be a consequence of the very existence of trajectories (as does the fact that in a two dimensional world there would be many more possibilities than just bosons and fermions) [14.

Bohmian mechanics is a fully deterministic theory of particles in motion, but a motion of a profoundly nonclassical, non-Newtonian sort. We should remark, however, that in the limit $\frac{\hbar}{m} \rightarrow 0$, the Bohm motion $Q_{t}$ approaches the classical motion.

But what does this theory, Bohmian mechanics, have to do with orthodox quantum theory, i.e., with the quantum formalism? Well, of course, they share Schrödinger's equation. However, in orthodox quantum theory noncommuting observables, represented by self-adjoint operators, play a fundamental role, while they do not appear at all in the formulation of Bohmian mechanics. Nonetheless, it can be shown that Bohmian mechanics not only accounts for quantum phenomena - this was essentially done by Bohm in 1952 and 1953 - but also embodies the quantum formalism itself, self-adjoint opera- tors, randomness given by $\rho=|\psi|^{2}$, and all the rest, as the very expression of its empirical import [1, 15.

Equations (2) (together with (3)) and (11) form a complete specification of the theory. There is no need, and indeed no room, for any further axioms. As for the status of the the familiar distribution $\rho=|\psi|^{2}$ in Bohmian mechanics, an answer is provided by reflecting upon the role of equilibrium measures for dynamical systems. Suppose one is interested in aspects of, say, the long time behavior, of patterns of statistical regularities which occur. Then some of the most basic of such information is usually provided by a measure stationary for the dynamics, so finding such a measure is often the key step in the analysis. Now it turns out that for Bohmian mechanics there is, in fact, no useful stationary measure, since the velocity field is typically time-dependent. Yet, $|\psi|^{2}$ is as good as a stationary measure. This distribution is in fact equivariant:

Consider an arbitrary initial ensemble $\rho$ and let

$$
\rho \rightarrow \rho_{t}
$$

be the ensemble evolution arising from Bohmian motion. If $\rho=\rho^{\psi}$ is a functional of $\psi$ we may also consider the ensemble evolutions arising from Schrödinger's equation

$$
\rho^{\psi} \rightarrow \rho^{\psi_{t}}
$$

$\rho^{\psi}$ is equivariant if these evolution are compatible

$$
\left(\rho^{\psi}\right)_{t}=\rho^{\psi_{t}}
$$

That $\rho=|\psi|^{2}$ is equivariant follows from comparing the quantum flux equation

$$
\frac{\partial|\psi|^{2}}{\partial t}+\operatorname{div} J^{\psi}=0
$$

where $J^{\psi}=\left(\mathbf{J}_{1}^{\psi} \ldots \mathbf{J}_{N}^{\psi}\right), \quad \mathbf{J}_{k}^{\psi}=\frac{\hbar}{m_{k}} \operatorname{Im}\left(\psi^{*} \nabla_{k} \psi\right)$, with the continuity equation associated with particle motion

$$
\frac{\partial \rho}{\partial t}+\operatorname{div}\left(\rho v^{\psi}\right)=0
$$

Since $J^{\psi}=v^{\psi}|\psi|^{2}$, the continuity equation is satisfied for $\rho=|\psi|^{2}$. Thus:

$$
\begin{aligned}
& \text { If } \rho\left(q, t_{0}\right)=\left|\psi\left(q, t_{0}\right)\right|^{2} \text { at some time } t_{0} \text { then } \\
& \rho(q, t)=|\psi(q, t)|^{2} \text { for all } t .
\end{aligned}
$$

Suppose now that a system has wave function $\psi$. We shall call the probability distribution on configuration space given by $\rho=|\psi|^{2}$ the quantum equilibrium distribution. And we shall say that a system is in quantum equilibrium when its configuration are randomly distributed according to the quantum equilibrium distribution. The empirical implications of Bohmian mechanics are based on the following 
Quantum equilibrium hypothesis (QEH): When a system has wave function $\psi$, the distribution $\rho$ of its configuration satisfies $\rho=$ $|\psi|^{2}$.

\section{EXISTENCE OF QUANTUM TRAJECTORIES}

Before proceeding to a sketch of how Bohmian mechanics accounts for quantum phenomena, we shall address the problem of whether Bohmian mechanics is a mathematically sound theory. After all, the velocity field (3) reveals rather obviously possible catastrophic events for the motion: $v^{\psi}$ is singular at the nodes of $\psi$, i.e., at points where $\psi=0$. We shall consider then the defining equations of Bohmian mechanics

$$
\begin{aligned}
\frac{d Q}{d t} & =v^{\psi}(Q) \\
i \hbar \frac{\partial \psi}{\partial t} & =H \psi,
\end{aligned}
$$

where $v^{\psi}$ is given by (3) and $H \psi$ is the right hand side of (1), and inquire about the existence and uniqueness of their solutions.

The "problem of the existence of dynamics" for Schrödinger's equation is usually reduced to showing that the relevant Hamiltonian $H$ (given by the particular choice of the potential $V$ ) is self-adjoint. This has been done in great generality, independent of the number of particles and for large classes of potentials, including singular potentials like the Coulomb potential, which is of primary physical interest 16, 17. In Bohmian mechanics we have not only Schrödinger's equation to consider but also the differential equation governing the motion of the particles. Thus the question of existence of the dynamics of Bohmian mechanics depends now on detailed regularity properties of the velocity field $v^{\psi}$. Local existence and uniqueness of Bohmian trajectories is guaranteed if the velocity field $v^{\psi}$ is locally Lipschitz continuous. We therefore certainly need greater regularity for the wave function $\psi$ than merely that $\psi$ be in $L^{2}$. Global existence is more delicate: In addition to the nodes of $\psi$, there are singularities comparable to those of Newtonian mechanics. Firstly,

even for a globally smooth velocity field the solution $Q_{t}$ may explode, i.e., it may reach infinity in finite time. Secondly, the singular points of the potential, are reflected in singular behavior of the wave function at such points, giving rise to singularities in the velocity field. (For example, the ground state wave function of one particle in a Coulomb potential $V(q)=1 /|q|, q \in \mathbb{R}^{3}$ ("hydrogen atom") has the form $e^{-|q|}$, which is not differentiable at the point $q=0$ of the potential singularity.)

The problem is then the following: Suppose that at some arbitrary "initial time" $\left(t_{0}=0\right)$ the $N$-particle configuration lies in the complement of the set of nodes and singularities of $\psi_{0}$. Does the trajectory develop in a finite amount of time into a singularity of the velocity field $v^{\psi}$, or does it reach infinity in finite time? It turns out that the answer is negative for "typical" initial values and a large class of potentials, including the physically most interesting case of $N$-particle Coulomb interaction with arbitrary charges and masses. Our results 18 20 are summarized by the following

Proposition 1 For a large class of Hamiltonians (including Coulomb with arbitrary charges and masses) and sufficiently regular initial datum $\psi_{0}$ the solution exists uniquely and globally in time for $\left|\psi_{0}\right|^{2}$-almost all initial configurations $Q_{0}$.

The quantity of central importance for our proof 20] of this theorem turns out to be the quantum current $j^{\psi}=\left(J^{\psi},|\psi|^{2}\right)$, with $J^{\psi}=v^{\psi}|\psi|^{2}$ the quantum probability flux. The absolute value of the flux through any surface in configuration-space-time controls the probability that a trajectory crosses that surface. Consider a smooth surface $\Sigma$ in configuration-space-time. The expected number of crossings of $\Sigma$ by the random trajectory $Q_{t}$ is given by

$$
\int_{\Sigma}\left|j_{t}(q) \cdot n\right| d \sigma
$$

where $n$ denotes the local unit normal vector at $(q, t)$. $\left(\int_{\Sigma}(j \cdot n) d \sigma\right.$ is the expected number of signed crossings. $)$ To get a handle on this consider first a small surface element which the trajectories cross at most once. The density of crossings is readily calculated to be $|j \cdot n|$. Invoking the linearity of the expectation value yields then the general statement. (In this regard we note that for the related problem in stochastic mechanics [12] the particle trajectories are realizations of a diffusion process and are hence not differentiable, i.e., velocities do not exist. Thus in stochastic mechanics the current does not have the same probabilistic significance and our analysis does not apply to stochastic mechanics.) Surfaces relevant to our analysis are those formed by the boundaries of neighborhoods around all the singular points for Bohmian mechanics. Loosely speaking, the importance of the quantum flux is grounded in the insight: "If there is no absolute flux into the singular points, the singular points are not reached."

\section{EMPIRICAL IMPLICATIONS}

A systematic analysis of the empirical implications of Bohmian mechanics falls naturally into two parts:

(A) The emergence and significance of other (nonconfigurational) observables.

(B) The clarification and justification of the QEH. 
As for (B), compare the QEH with the Gibbs postulate (GP) of statistical mechanics:

$$
\begin{gathered}
\text { quantum equilibrium } \quad \rho=|\psi|^{2} \\
\text { thermodynamic equilibrium } \quad \rho \sim e^{-\beta H}
\end{gathered}
$$

While the complete justification of the GP is remarkably difficult (and as of now is nonexistent), that of the QEH is relatively easy [1].

As for (A), the crucial observation has been made by Bell [21]:

... in physics the only observations we must consider are position observations, if only the positions of instrument pointers. It is a great merit of the de Broglie-Bohm picture to force us to consider this fact. If you make axioms rather than definitions and theorems about the 'measurement' of anything else then you commit redundancy and risk inconsistency.

When one comes to "measurements" and "observables," a warning against the misuse of these words is mandatory. We again quote Bell [22]:

... The first charge against "measurement," in the fundamental axioms of quantum mechanics, is that it anchors the shifty split of the world into "system" and "apparatus." A second charge is that the word comes loaded with meaning from everyday life, meaning which is entirely inappropriate in the quantum context. When it is said that something is "measured" it is difficult not to think of the result as referring to some preexisting property of the object in question. This is to disregard Bohr's insistence that in quantum phenomena the apparatus as well as the system is essentially involved. ... Even in a lowbrow practical account, I think it would be good to replace the word "measurement," in the formulation, with the word "experiment."

\section{A. Experiments}

When we speak of a very general experiment $\mathcal{E}$, beginning, say, at $t=0$ and ending at time $T$, we have in mind a fairly definite initial state $\Phi_{0}=\Phi_{0}(y)$ of the apparatus, one for which the apparatus should function as intended, as well as a definite initial state of the system $\psi=\psi(x)$ on which the experiment is performed. Under these conditions it turns out that the composite system formed by system and apparatus, with generic configuration $q=(x, y)$, has initial wave function:

$$
\Psi_{0}=\psi \otimes \Phi_{0} .
$$

Moreover, $\mathcal{E}$ will be specified by a unitary operator $U$ generating the time evolution arising from the interaction of the system and apparatus, which yields the wave function $\Psi_{T}$ of the composite system after the experiment; and a calibration function $F$ from the configuration space of the composite system to some value space, e.g. $\mathbb{R}$, fixing the scale of the experiment, and defining the result $\mathbf{Z} \equiv F\left(Q_{T}\right)$ of the experiment-think of the "orientation of the apparatus pointer" or some coarsegraining thereof - as a function of the configuration $Q_{T}$ of the system and apparatus after the experiment.

Assume QEH. Then $Q_{T}$ is randomly distributed according to the quantum equilibrium measure $\mathbb{P}_{T}(d q)=$ $\left|\Psi_{T}\right|^{2} d q$ and $\mathrm{Z}$ is a random variable (on the probability space of the initial configurations of system and apparatus) with distribution given by the probability measure

$$
\mu=\mathbb{P}_{T} \circ F^{-1} .
$$

A stepping stone of our analysis [15] is the following

Proposition 2 With any experiment $\mathcal{E}$ there is always associated a positive-operator-valued measure (POV) $O(d \lambda)$ such that

$$
\mu(d \lambda)=\langle\psi, O(d \lambda) \psi\rangle
$$

This follows very easily from the observation that the map $\psi \rightarrow \mu$ from (initial system) wave functions to probability measures on the value space, explicitly given by the following sequence of maps

$$
\psi \rightarrow \Psi=\psi \otimes \Phi_{0} \rightarrow \Psi_{T} \rightarrow \mathbb{P}_{T} \rightarrow \mu=\mathbb{P}_{T} \circ F^{-1},
$$

is a normalized bilinear map on the system Hilbert space $\mathcal{H}$, since the middle map to the quantum equilibrium distribution,

$$
\Psi_{T} \rightarrow \mathbb{P}_{T}(d q)=\Psi_{T}^{*} \Psi_{T} d q,
$$

is obviously bilinear, while all the other maps are linear, all but the second trivially so. Now, by elementary functional analysis, the notion of such a bilinear map is completely equivalent to that of a POV! We note that when the experiment is "measurement-like" (by this we merely mean that, unlike a coin flip, the outcome is reproducible) the POV $O$ is actually a projection valued measure (PV) and with every such experiment we may associate a self-adjoint operator $A$,

$$
\mathcal{E} \rightarrow A,
$$

which governs the statistics of the outcomes in the usual way 115$]$.

We recall that because of difficulties in the application of the usual operator formalism, it has been proposed in the framework of the so called operational approach to 
quantum mechanics that we should go beyond operatorsas-observables, to "generalized observables" 23, 26. The basis of this extension lies in the observation that, by the spectral theorem, the concept of self-adjoint operator is completely equivalent to that of (normalized) projectionvalued measure $(\mathrm{PV})$ on the value space $\mathbb{R}$. Since orthogonal projections are among the simplest examples of positive operators, a natural generalization of a "quantum observable" is then provided by a (normalized) positiveoperator-valued measure (POV) - when a POV is sandwiched by a wave function it generates a probability distribution.

On the other hand, the emergence and role of POV's in Bohmian mechanics is not a matter of generalization; rather it is merely an expression of the bilinearity of quantum equilibrium together with the linearity of Schrödinger's evolution. Thus the fact that with $e v$ ery experiment is associated a POV, which forms a compact expression of the statistics for the possible results, is a near mathematical triviality. It is therefore rather dubious that the occurrence of POV's as observablesthe simplest case of which is that of PV's - can be regarded as suggesting any deep truths about reality or about epistemology. In particular, so understood, the notion of self-adjoint-operator-as-observable $A$ in no way implies that anything is really being measured in the experiment with which $A$ is associated, and certainly not the operator $A$ itself! In a general experiment no property is being measured, even if the experiment happens to be measurement-like. (In this regard we note that experiments associated with the position operator are for the most part an important exception, though there are "measurements" of the position operator that are not measurements of the actual position 27 29,15.)

That self-adjoint operators are associated only with special experiments is a further indication that the usual quantum formalism, based only on self-adjoint operators, is merely an idealization, rarely directly relevant in practice. Indeed, a great many significant real-world experiments are simply not at all associated with operators in the usual way $30,31,15,32$.

Consider for example an electron with fairly general initial wave function, and surround the electron with a "photographic" plate, away from (the support of the wave function of) the electron, but not too far away. This set-up measures the position of "escape" of the electron from the region surrounded by the plate. Notice that since in general there is no definite time of escape, it is not at all clear which operator should correspond to the escape position. Indeed, it can be shown [31,15] that there is no such operator, that for the experiment just described the probabilities for the possible results cannot be expressed in the usual form, and in fact are not given by the spectral measure for any operator.

We note that the study of the asymptotic limit for this situation - the scattering regime - is the starting point for a reformulation of scattering theory [31] based on the so called scattering-into-cones-theorem, proved by Dollard [33], and the flux-across-surfaces-theorem 34, of which a complete proof is still lacking.

\section{B. Quantum Equilibrium}

We'd like now to turn to the clarification and justification of the QEH [1,8,7]. There are some crucial subtleties in the $\mathrm{QEH}$, which we can begin to appreciate by first asking the question: Which systems should be governed by Bohmian mechanics? The systems which we normally consider are subsystems of a larger system-for example, the universe - whose behavior (the behavior of the whole) determines the behavior of its subsystems (the behavior of the parts). Thus for a Bohmian universe, it is only the universe itself which a priori-i.e., without further analysis - can be said to be governed by Bohmian mechanics. So let's consider such a universe. Our first difficulty immediately emerges: In practice $\rho=|\psi|^{2}$ is applied to (small) subsystems. But only the universe has been assigned a wave function (which we shall now denote by $\Psi)$ ! What is meant then by the RHS of $\rho=|\psi|^{2}$, i.e., by the wave function of a subsystem?

Let's go further. Fix an initial wave function $\Psi_{0}$ for this universe. Then since the Bohmian evolution is completely deterministic, once the initial configuration $Q$ of this universe is also specified, all future events, including of course the results of measurements, are determined. Now let $X$ be some subsystem variable - say the configuration of the subsystem at some time $t$ - which we would like to be governed by $\rho=|\psi|^{2}$. But how can this possibly be, when there is nothing at all random about $X$ ?

Of course, if we allow the initial universal configuration $Q$ to be random, distributed according to the quantum equilibrium distribution $\left|\Psi_{0}(Q)\right|^{2}$, it follows from equivariance that the universal configuration $Q_{t}$ at later times will also be random, with distribution given by $\left|\Psi_{t}\right|^{2}$, from which you might well imagine that it follows that any variable of interest, e.g., $X$, has the "right" distribution. But even if this is so (and it is), it would be devoid of physical significance! What possible physical significance can be assigned to an ensemble of universes, when we have but one universe at our disposal, the one in which we happen to reside? We cannot perform the very same experiment more than once. But we can perform many similar experiments, differing, however, at the very least, by location or time. In other words, insofar as the use of probability in physics is concerned, what is relevant is not sampling across an ensemble of universes, but sampling across space and time within a single universe. What is relevant is empirical distributions - actual relative frequencies for an ensemble of actual events. 
Two problems must thus be addressed, that of the meaning of the wave function $\psi$ of a subsystem and that of randomness. It turns out that once we come to grips with the first problem, the question of randomness almost answers itself. We obtain just what we want - that $\rho=|\psi|^{2}$ in the sense of empirical distributions; we find that in a typical Bohmian universe an appearance of randomness emerges, precisely as described by the quantum formalism.

What about the wave function of a subsystem? Given a subsystem we may write $q=(x, y)$ where $x$ and $y$ are generic variables for the configurations of the subsystem and its environment. Similarly, we have $Q_{t}=(X, Y)$ for the actual configurations (at time $t$ ). What is the simplest possibility for the wave function of the subsystem, the $x$-system; what is the simplest function of $x$ which can sensibly be constructed from the actual state of the universe at time $t$ (which we remind you is given by $Q_{t}$ and $\left.\Psi_{t}=\Psi\right)$ ? Clearly the answer is what we call the conditional wave function

$$
\psi(x)=\Psi(x, Y)
$$

This is all we need! (This is not quite the right notion for the "effective" wave function of a subsystem, upon which we shall elaborate in the next section, but whenever the latter exists it agrees with what we have just described.) Now see what you can do without actual configurations! (You'll, of course, quickly encounter the measurement problem!)

The main result of our analysis [1] is summarized by the following

Proposition 3 When a system has wave function $\psi$, the distribution $\rho$ of its configuration typically satisfies $\rho=$ $|\psi|^{2}$.

This means that for typical initial configurations of the universe, the empirical distribution of an ensemble of $M$ identical subsystems with wave function $\psi$ converges to $\rho=|\psi|^{2}$ for large $M$. The statement refers to an equal-time ensemble or to a multi-time ensemble and the notion of typicality is expressed by the measure $\mathbb{P}^{\Psi_{0}}(d Q)$ and more importantly by the conditional measure $\mathbb{P}^{\Psi_{0}}(d Q \mid \mathcal{M})$, where the set $\mathcal{M}$ takes into account any kind of prior information - always presentreflecting the macroscopic state at a time prior to all experiments. Moreover, the above proposition holds under physically minimal conditions, expressed by certain measurability conditions reflecting the requirement that facts about results and initial experimental conditions not be forgotten.

\section{THE EFFECTIVE WAVE FUNCTION}

Let's pause for a moment and get familiar with the notion of conditional wave function by looking at a very simple example:

Consider two particles in one dimension, whose evolution is governed by the Hamiltonian

$H=H^{(x)}+H^{(y)}+H^{(x y)}=-\frac{\hbar^{2}}{2 m}\left(\frac{\partial^{2}}{\partial x^{2}}+\frac{\partial^{2}}{\partial y^{2}}\right)+\frac{1}{2} \kappa(x-y)^{2}$.

For simplicity let us set $\hbar=m=\kappa=1$. Assume that the composite has initial wave function

$$
\Psi_{0}=\psi \otimes \Phi_{0}
$$

with $\quad \psi(x)=\pi^{-\frac{1}{4}} e^{-\frac{x^{2}}{2}} \quad$ and $\quad \Phi_{0}(y)=\pi^{-\frac{1}{4}} e^{-\frac{y^{2}}{2}}$.

By solving the basic equations of Bohmian mechanics one easily obtains that

$$
\Psi_{t}(x, y)=\pi^{-\frac{1}{2}}(1+i t)^{-\frac{1}{2}} e^{-\frac{1}{4}\left[(x-y)^{2}+\frac{(x+y)^{2}}{1+2 i t}\right]},
$$

and

$$
X_{t}=a(t) X+b(t) Y \quad \text { and } \quad Y_{t}=b(t) X+a(t) Y,
$$

where $a(t)=\frac{1}{2}\left[\left(1+t^{2}\right)^{\frac{1}{2}}+1\right], b(t)=\frac{1}{2}\left[\left(1+t^{2}\right)^{\frac{1}{2}}-1\right]$, and $X, Y$ are the initial conditions of the two particles. Focus now on one of the two particles (the $x$-system) and regard the other one as its environment (the $y$-system). The conditional wave function of the $x$-system

$$
\psi_{t}(x)=\Psi_{t}\left(x, Y_{t}\right)
$$

depends, through $Y_{t}$, on both the initial conditions for the environment and the initial condition for the particle. In other words, the evolution of $\psi_{t}$ is random, with probability law determined by $\left|\Psi_{0}\right|^{2}$. In particular, $\psi_{t}$ does not satisfy Schrödinger's equation for any $H^{(x)}$.

We remark that even when the $x$-system is dynamically decoupled from its environment, the conditional wave function will not in general evolve according to Schrödinger's equation. Thus the conditional wave function lacks the dynamical implications from which the wave function of a system derives much of its physical significance. These are, however, captured by the notion of effective wave function:

Suppose that

$$
\Psi(x, y)=\psi(x) \Phi(y)+\Psi^{\perp}(x, y)
$$

where $\Phi$ and $\Psi^{\perp}$ have macroscopically disjoint $y$ supports. If

$$
Y \in \operatorname{supp} \Phi
$$


we say that $\psi$ is the effective wave function of the $x$-system. Of course, $\psi$ is also the conditional wave function-nonvanishing scalar multiples of wave functions are naturally identified. (In fact, in Bohmian mechanics the wave function is naturally a projective object since wave functions differing by a multiplicative constant - possibly time-dependent - are associated with the same vector field, and thus generate the same dynamics.)

One might wonder why systems ever possess an effective wave function. In fact, in general they don't! For example the $x$-system will not have an effective wave function when, for example, it belongs to a larger microscopic system whose effective wave function doesn't factorize in the appropriate way. However, the larger the environment of the $x$-system, the greater is the potential for the existence of an effective wave function for this system, owing in effect to the abundance of "measurement-like" interactions with a larger environment. The notion of effective wave function is robust, as there is a natural tendency toward the formation of stable effective wave functions via dissipation: Suppose that initially the $y$ supports of $\Phi$ and $\Psi^{\perp}$ are just "sufficiently" (but not macroscopically) disjoint; then, due to the interactions with the environment, the amount of $y$-disjointness will tend to increase dramatically as time goes on, with, as in a chain reaction, more and more degrees of freedom participating in this disjointness. When the effect of this dissipation, or "decoherence," are taken into account, one find that even a small amount of $y$-disjointness will often tend to become "sufficient," and quickly "more than sufficient," and finally macroscopic.

The ever-decreasing possibility of interference between macroscopically distinct wave functions due to typically uncontrollable interactions with the environment is nowadays often referred to as decoherence (Griffiths [35], Omnes [36], Leggett [37, Zurek [38], Joos-Zeh [39]) and has been regarded (Gell-Mann-Hartle 40]) as a crucial ingredient for extracting a "quasiclassical domain of familiar experience" from the quantum formalism itself (see also 41]). One of the best descriptions of the mechanism of decoherence, though not the word itself, can be found in the Bohm's 1952 "hidden variables" paper [2]. We wish to emphasize, however, as did Bell in his article "Against Measurement" 22], that decoherence in no way comes to grips with the measurement problem itself, being merely a necessary, but not a sufficient, condition for its complete resolution. In contrast, the very notion of effective wave function resolves the measurement problem at once.

Consider for example an experiment $\mathcal{E}$ with an apparatus so designed that there are only finitely (or countably) many possible outcomes, labeled by $\alpha \in I$. Then, after the experiment the wave function of the composite is of the form

$$
\Psi_{T}=\sum_{\alpha \in I} \psi_{\alpha} \otimes \Phi_{\alpha}
$$

where the $\Phi_{\alpha}$ are (normalized) apparatus states supported by the macroscopically distinct sets $\alpha \in I$ of apparatus configurations. Of course, for Bohmian mechanics, the terms of (6) are not all on the same footing: one of them, and only one, is selected, or more precisely supported, by the outcome - corresponding, say, to $\alpha_{0}$ which actually occurs. It follows that after the experiment, at time $T$, the $x$-system has effective wave function $\psi_{\alpha_{0}}$. This is how collapse (or reduction) of the effective wave function to the one associated with the outcome $\alpha_{0}$ arises in Bohmian mechanics.

Note that while in orthodox quantum theory the collapse is merely superimposed upon the unitary evolution - without a precise specification of the circumstances under which it may legitimately be invoked-we have now, in Bohmian mechanics, that the evolution of the effective wave function is actually given by a stochastic process, which consistently embodies both unitarity and collapse as appropriate. In particular, the effective wave function of a subsystem evolves according to Schrödinger's equation when this system is suitably isolated. Otherwise it "pops in and out" of existence in a random fashion, in a way determined by the continuous (but still random) evolution $\psi_{t}$ of the conditional wave function. (In this regard, as far as the general problem of chaotic behavior in quantum theory is concerned, note that there is nothing in Bohmian mechanics which would preclude sensitive dependence on initial conditions, of $Q_{t}$ on $Q_{0}$ and $\psi_{0}$, and hence positive Lyapunov exponents. In Bohmian mechanics "quantum chaos" arises, as in the classical case, solely from the dynamical law and not from the collapse rule applied in measurements 42 .)

\section{QUANTUM PHYSICS WITHOUT QUANTUM PHILOSOPHY}

We would like to make a few comments now about Bohmian mechanics and "the real world." There is at best an uneasy truce between orthodox quantum theory and the view that there is an objective reality, of a more or less familiar sort on the macroscopic level. Recall, for example, Schrödinger's cat. What does Bohmian mechanics contribute here? In a word, everything! A world of objects, of large collections of particles which combine and move more or less as a whole, presents no conceptual difficulty for Bohmian mechanics, since Bohmian mechanics is after all a theory of particles in motion and allows for the possibility of such large collections.

So what, when all is said and done, does the incorporation of the particle positions, of the configurations, buy us? A great deal: 
1. randomness

2. familiar (macroscopic) reality

3. the wave function of a (sub)system

4. collapse of the wave packet

5. absolute uncertainty

We have not yet explicitly addressed item 5.5 is a consequence of the analysis of $\rho=|\psi|^{2}$. It expresses the impossibility of obtaining information about positions more detailed than what is given by the quantum equilibrium distribution. It provides a precise, sharp foundation for the uncertainty principle, and is itself an expression of global quantum equilibrium [1].

When all is said and done, Bohmian mechanics emerges as a precise and coherent "quantum theory" providing a microscopic foundation for the quantum formalism. To sum up, it seems fair to say that Bohmian mechanics is nothing but quantum physics without quantum philosophy. Moreover, the only objections which are usually raised against Bohmian mechanics are merely philosophical. Now we don't wish to enter here into philosophical disputes. We would, however, like to mention that in response to the outrage sometimes expressed towards the suggestion that particles might have positions when they are not, or cannot be, observed, Bell, referring to theories such as Bohm's, has said that

Absurdly, such theories are known as "hidden variable" theories. Absurdly, for there it is not in the wave function that one finds an image of the visible world, and the results of experiments, but in the complementary "hidden"(!) variables. Of course the extra variables are not confined to the visible "macroscopic" scale. For no sharp definition of such a scale could be made. The "microscopic" aspect of the complementary variables is indeed hidden from us. But to admit things not visible to the gross creatures that we are is, in my opinion, to show a decent humility, and not just a lamentable addiction to metaphysics 43.

\section{ACKNOWLEDGMENTS}

This work was supported by the DFG, by NSF Grant No. DMS-9305930, and by INFN.
[1] D. Dürr, S. Goldstein, and N. Zanghì. Quantum Equilibrium and the Origin of Absolute Uncertainty. Journal of Statistical Physics, 67:843-907, 1992.

[2] D. Bohm. A suggested interpretation of the quantum theory in terms of "hidden" variables: Part I. Physical Review, 85:166-179, 1952. Reprinted in 44.

[3] D. Bohm. A suggested interpretation of quantum theory in terms of "hidden variables": Part II. Physical Review, 85:180-193, 1952. Reprinted in 44.

[4] D. Bohm. Proof that probability density approaches $|\psi|^{2}$ in causal interpretation of quantum theory. Physical Review, 89:458-466, 1953.

[5] D. Bohm and B. J. Hiley. The Uundivided Universe: An Ontological Intepretation of Quantum Theory. Routledge \& Kegan Paul, London, 1993.

[6] J. S. Bell. Speakable and unspeakable in quantum mechanics. Cambridge University Press, Cambridge, 1987.

[7] D. Dürr, S. Goldstein, and N. Zanghì. A Global Equilibrium as the Foundation of Quantum Randomness. Foundations of Physics, 23:721-738, 1993.

[8] D. Dürr, S. Goldstein, and N. Zanghì. Quantum Mechanics, Randomness, and Deterministic Reality. Physics Letters A, 172:6-12, 1992.

[9] P. R. Holland. The Quantum Theory of Motion. Cambridge University Press, Cambridge, 1993.

[10] D. Z. Albert. Bohm's alternative to quantum mechanics. Scientific American, 270:32-39, May 1994.

[11] J. S. Bell. On the problem of hidden variables in quantum mechanics. Reviews of Modern Physics, 38:447-452, 1966. Reprinted in [44] and in [6].

[12] E. Nelson. Quantum Fluctuations. Princeton University Press, Princeton, N.J., 1985.

[13] S. Goldstein. Stochastic mechanics and quantum theory. Journal of Statistical Physics, 47:645-667, 1987.

[14] D. Dürr, S. Goldstein, and N. Zanghì. Bohmian mechanics, identical particles, parastatistics, and anyons. In preparation, 1994.

[15] M. Daumer, D. Dürr, S. Goldstein, and N. Zanghì. On the role of operators in quantum theory. In preparation, 1994.

[16] T. Kato. Fundamental properties of Hamiltonian operators of Schrödinger type. Trans. Am. Math. Soc., 70:195211, 1951.

[17] M. Reed and B. Simon. Methods of Modern Mathematical Physics II. Academic Press, New York, 1975.

[18] K. Berndl, D. Dürr, S. Goldstein, G. Peruzzi, and N. Zanghì. Existence of Trajectories for Bohmian Mechanics. International Journal of Theoretical Physics, 32:2245-2251, 1993.

[19] K. Berndl, D. Dürr, S. Goldstein, and N. Zanghì. Selfadjointness and the Existence of Deterministic Trajectories in Quantum Theory. In On Three Levels: The Micro-, Meso-, and Macroscopic Approaches in Physics, (NATO ASI Series B: Physics, Volume 324, Plenum, New York, 1994) pp. 429-434.

[20] K. Berndl, D. Dürr, S. Goldstein, G. Peruzzi, and N. Zanghì. On the global existence of Bohmian mechanics. Comm. Math. Phys., to appear.

[21] J. S. Bell. On the impossible pilot wave. Foundations of Physics, 12:989-999, 1982. Reprinted in [6].

[22] J. S. Bell. Against "measurement". Physics World, 3:33- 
40, 1990. Also in 45].

[23] D. Davies. Quantum Theory of Open Systems. Academic Press, London-New York-San Francisco, 1976.

[24] A. S. Holevo. Probabilistic and Statistical Aspects of Quantum Theory, Volume 1 of North-Holland Series in Statistics and Probability. North-Holland, AmsterdamNew York-Oxford, 1982.

[25] K. Kraus. States, Effects, and Operations. Lectures Notes in Physics, 190, 1983.

[26] G. Ludwig. Foundations of Quantum Mechanics, Volume 1. Springer, Heidelberg-Berlin-New York, 1983.

[27] B. Englert, M. D. Scully, G. Süssman, and H. Walther. Surrealistic Bohm Trajectories. Z.f.Naturforschung, 47a:1175-1186, 1992

[28] D. Dürr, W. Fusseder, S. Goldstein, and N. Zanghì. Comment on: Surrealistic Bohm Trajectories. Z.f.Naturforschung, 48a:1261-1262, 1993.

[29] C. Dewdney, L. Hardy, and E. J. Squires. How late measurements of quantum trajectories can fool a detector. Phys. Lett. A, 184:6-11, 1993.

[30] M. Daumer and S. Goldstein. Observables, measurements and phase operators from a Bohmian perspective. In Proceedings of the Second International Workshop on Squeezed States and Uncertainty Relations, Han, Kim, and Man'ko (eds.), NASA Conference Publication No. 3219, 231, 1993.

[31] M. Daumer, D. Dürr, S. Goldstein, and N. Zanghì. Scattering and the Role of Operators in Bohmian Mechanics. In On Three Levels: The Micro-, Meso-, and Macroscopic Approaches in Physics, (NATO ASI Series B: Physics, Volume 324, Plenum, New York, 1994).

[32] C.R. Leavens. Arrival time distribution. Physics Letters A, 178:27-32, 1993.

[33] J.D. Dollard. Scattering into cones. Communications in Mathematical Physics, 12:193, 1969.

[34] M. Combes, R. G. Newton, and R. Shtokhamer. Scattering into cones and flux across surfaces. Physical Review D, 11:366, 1975 .

[35] R. B. Griffiths. Consistent histories and the interpretation of quantum mechanics. Journal of Statistical Physics, 36:219-272, 1984.

[36] R. Omnes. Logical reformulation of quantum mechanics. Journal of Statistical Physics, 53:893-932, 1988.

[37] A. J. Leggett. Macroscopic quantum systems and the quantum theory of measurement. Supplement of the Progress of Theoretical Physics, 69:80-100, 1980.

[38] W. H. Zurek. Environment-induced superselection rules. Physical Review D, 26:1862-1880, 1982.

[39] E. Joos and H. D. Zeh. The emergence of classical properties through interaction with the environment. Zeitschrift für Physik B, 59:223-243, 1985.

[40] M. Gell-Mann and J. B. Hartle. Quantum mechanics in the light of quantum cosmology. In W. Zurek, editor, Complexity, Entropy, and the Physics of Information, pages 425-458. Addison-Wesley, Reading, 1990. Also in 46.

[41] S. Goldstein and D. N. Page. Linearly positive histories. Preprint, 1994.

[42] D. Dürr, S. Goldstein, and N. Zanghì. Quantum Chaos, Classical Randomness and Bohmian Mechanics. Journal of Statistical Physics, 68:259-270, 1992.
[43] J. S. Bell. Are there quantum jumps? In C. W. Kilmister, editor, Schrödinger. Centenary celebration of a polymath. Cambridge University Press, Cambridge, 1987. Reprinted in [6].

[44] J. A. Wheeler and W. H. Zurek. Quantum Theory and Measurement. Princeton University Press, Princeton, N.J., 1983.

[45] A. I. Miller, editor. Sixty-two Years of Uncertainty: Historical, Philosophical, and Physical Inquiries into the Foundations of Quantum Mechanics, pages 17-31. Plenum Press, New York, 1990.

[46] S. Kobayashi, H. Ezawa, Y. Murayama, and S. Nomura, editors. Proceedings of the 3rd International Symposium on Quantum Mechanics in the Light of New Technology. Physical Society of Japan, 1990. 